\title{
Sampling method for woody biomass particles conveyed by air in the fuel pipe of a pulverized coal firing boiler
}

\author{
Shohei MATSUNARI*, Kazuhiro WATANABE*, Emi OHNO* and Makoto ECHIZENYA* \\ ${ }^{*} \mathrm{IHI}$ Corporation \\ 3-1-1, Toyosu, Koto-ku, Tokyo 135-8710, Japan \\ E-mail: matsunari1005@ihi-g.com
}

Received: 5 March 2020; Revised: 24 April 2020; Accepted: 7 July 2020

\begin{abstract}
In Japan, a feed-in tariff was introduced to promote and expand the renewable energy ratio to $22-24 \%$ by FY 2030. Biomass is one of important renewable energy. Recently, several biomass projects for not only fluidized bed boilers, but also pulverized coal fired boilers, are being planned around the world to reduce $\mathrm{CO} 2$ emissions as the main reason. The required particle size of woody biomass for combustion in a pulverized coal fired boiler is around $1 \mathrm{~mm}$, while that of coal is several tens of $\mu \mathrm{m}$. Woody biomass particles grinded by a mill are sent to burners via a fuel pipe by air. It is important to measure the particle size distribution in order to understand combustibility and grindability. However, it was unknown whether the particle size distribution could be measured by the same sampling method for pulverized coal. We conducted sampling tests of woody biomass particles in a fuel pipe using the isokinetic sampling method. As a result, we observed that the flow of woody biomass particles in the pipe was significantly non-uniform to the influence of the upstream elbow. It is necessary for woody biomass to measure at a pitch of at least $45^{\circ}$ in order to obtain the particle size distribution in a fuel pipe.
\end{abstract}

Keywords : Woody biomass, Particle size distribution, Isokinetic sampling method, Pulverized coal fired boiler, Biomass co-firing

\section{Introduction}

In Japan, approximately $92 \%$ of energy is imported from overseas, therefore improving the energy self-sufficiency rate is a major issue. As such, in order to increase the renewable energy ratio to between 22 and 24\% by FY 2030, a feed-in tariff for renewable energy has been introduced(Agency for Natural Resources and Energy, 2018, 2020). Biomass is one form of renewable energy. In recent years, coal-fired power producers are beginning to convert all or part of their fuel to biomass. Even in Japanese pulverized coal-fired boilers, projects for co-firing woody biomass at a ratio of $30 \%$ or more are increasing (IHI Corporation, 2017). Woody biomass is easier to burn because it has more volatile matter than coal. The required particle size of woody biomass for combustion in pulverized coal-fired boilers is around $1 \mathrm{~mm}$, while that of coal is several tens of $\mu \mathrm{m}$. Therefore, woody pellets which consist of particles approximately $1 \mathrm{~mm}$ in size can easily be used as fuel.

A coal mill is retrofitted as a woody pellet mill, and the pellets are crushed and sent to the multi-burner via each fuel pipe by air in the same way as pulverized coal. If the particle size of the fuel sent to the burner is $1 \mathrm{~mm}$ or more, the combustibility in the boiler will deteriorate. Unbalance of particle size of fuel among burners from a mill makes it possible to deteriorate the combustibility of some burners. These are reasons why it is important to understand particle size distribution around $1 \mathrm{~mm}$ in the fuel pipe.

The particle size of crushed woody pellet is larger than that of pulverized coal. It was unclear whether the particle size distribution of crushed woody pellet could be measured using the measurement method used in the case of pulverized coal (ASME, 1991) (ASTM, 1946). Therefore, we collected samples of woody biomass particles in a fuel pipe using a method based on ASME, then investigated and examined how particle size distribution could be measured. 


\section{Experimental Section}

\subsection{Investigation items and experimental facility}

The four investigation items are as follows;

(1) Confirmation of distribution of woody biomass particles in the fuel pipe cross-section

In the fuel pipe cross-section, the positions of woody biomass particles of each size are confirmed. We also confirm the presence of non-uniform flow. Normally sample connections are arranged at $90^{\circ}$. However, in this test, we sampled at a pitch of $45^{\circ}$.

(2) Effect of sampling tube tip opening size

The particle size of crushed woody pellet is larger than pulverized coal. Using a conventional sampling tube, there is a possibility that coarse particles cannot be sampled because the tip size is smaller than woody biomass particle size. Evaluated the particle size distribution when the sampling tube tip is enlarged.

(3) Effect of sampling time

Flow condition in fuel pipe is unsteady. We changed sampling time and investigated the influence on the particle size distribution of the sampled woody biomass particles.

(4) Comparison of size distribution on arrangement of sampling station

The arrangements of sampling station are compared between equal intervals and at equal areas.

Schematic drawing of the test facility is shown in Fig.1. Woody biomass particles discharged from a mill are sent to the burner via a fuel pipe by air.

Woody biomass particles are sampled at sample connections placed on a fuel pipe. The sample connections are installed in two places on the vertical and horizontal pipe. The shape of the sample connections will be described in Section 2.3.

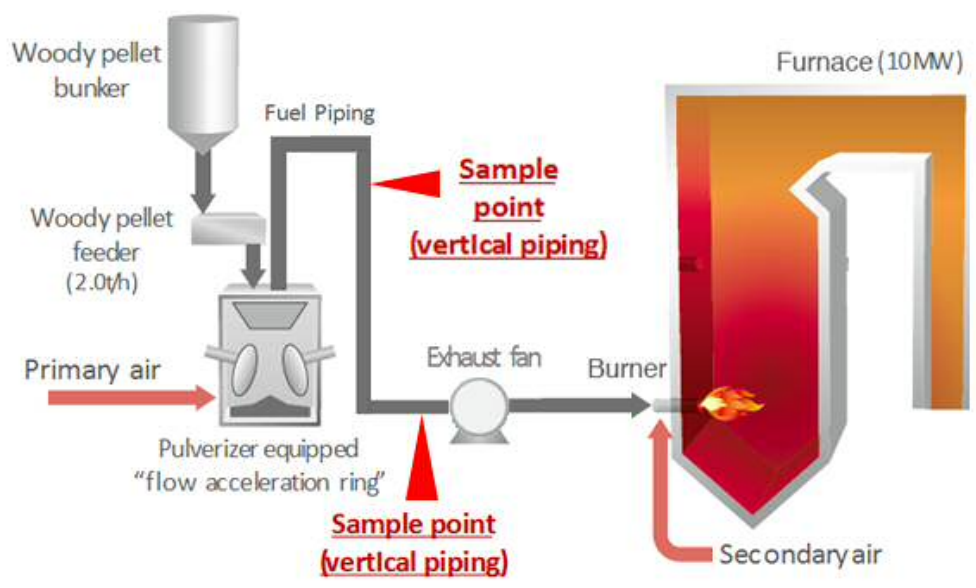

Fig. 1 Schematic drawing of experimental facility

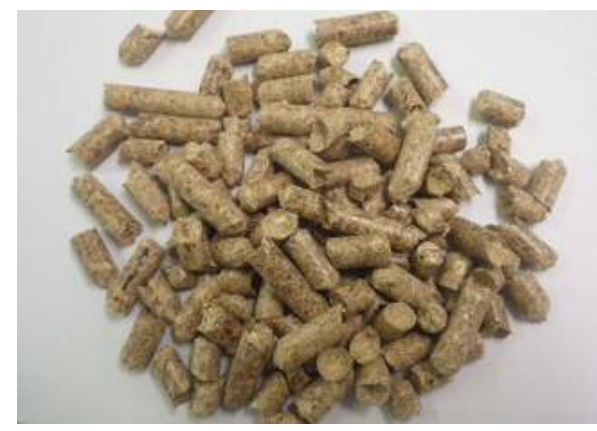

Fig. 2 Appearance of woody pellet

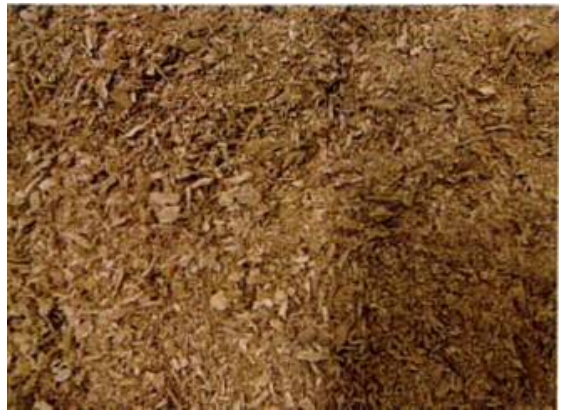

Fig. 3 Woody particle before pelletizing 


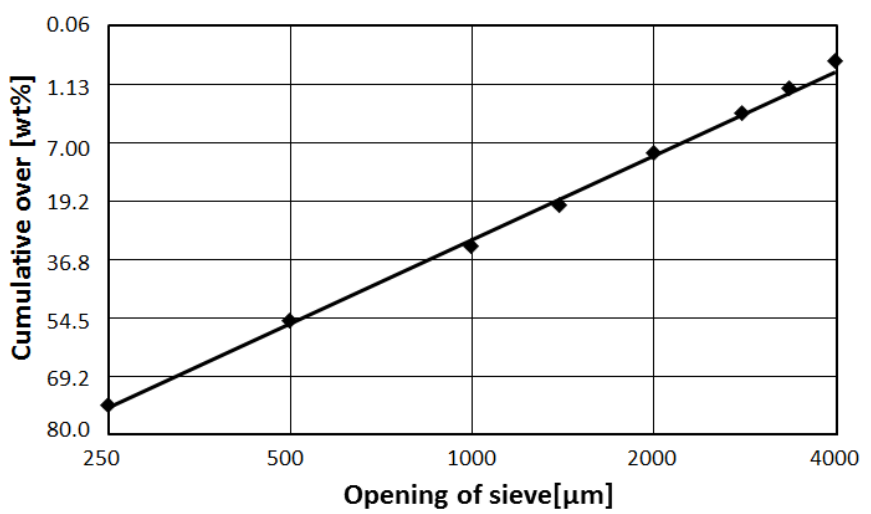

Fig. 4 Particle size distribution of particles before pelletizing on a Rosin-Rammler diagram

\subsection{Test material}

In order to confirm non-uniform flow of each woody biomass particles in a fuel pipe, woody pellet containing coarse particles with a major axis diameter of approximately $10 \mathrm{~mm}$. Fig. 2 shows the appearance of the pellet. The diameter of the pellet is $8.6-9.5 \mathrm{~mm}$ and the length is 4.6-6.0 mm. Fig. 3 shows Appearance of woody particle before pelletizing. Fig. 4 shows the particle size distribution of the woody particle before pelletizing on a Rosin-Rammler diagram. The pellets are made from Acasia.

\subsection{Pitch of sample connections}

Sampling points as shown in Fig. 5 are installed in the horizontal and vertical pipe. Four sample connections are installed at a pitch of $45^{\circ}$ along the outer periphery in the same cross-section of the pipe. The sampling tube is inserted into the sample connection and the tip of sampling tube is placed on the sampling station shown in Fig.7.

\subsection{Sampling station}

The sampling stations with two types of arrangements are shown in Fig. 7.

- Sampling station of equal areas (Fig. 7(a))

The cross-section of the pipe is divided into equal areas concentrically. The center of gravity of each area is taken as sampling station. The woody biomass particles sampled at the sampling station are representative of particles passing through the area.

- Sampling station of equal intervals (Fig. 7(b))

Sampling stations are arranged at equal intervals in order to cover as many areas as possible without equalizing the area.

\subsection{Sampling tube and sampling time}

As shown in Table 1, we used three types of sampling tubes. The tip opening dimension of each sampling tube is different. The diameter of the sampling tube is $\varphi 25.4$.Sampling tube No.1 is dimensioned based on ASME, and this is the size we use conventionally.

We changed the sampling time to $30,60,120,360$ sec. per sampling station and investigated the effect.

\subsection{Sampling procedure}

The below isokinetic sampling method was applied.

(1) Insert sampling tube into fuel piping through the sample connection, and place the center of the tip opening on the sampling station closest to the sample connection.

(2) The sampling station is placed at the location shown in Section 2.4, and sampling is performed for the time 
shown in Section 2.5.

(3) After the sampling time at one sampling station has passed, move the tip onto the next sampling station and sample again for the sampling time continuously.

(4) After sampling at all sampling stations in one sample connection is complete, pull out the sampling tube.

(5) Repeat the same procedure at the next sample connection.

If obtaining woody biomass particle distribution at one sampling station in Section 3.1, after sampling woody biomass particles at a single sampling station, pull the sampling tube out without moving to the next sampling station.

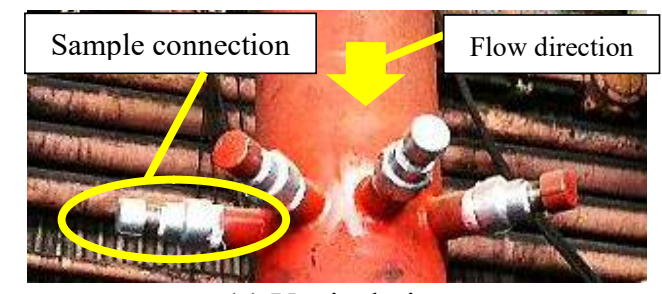

(a) Vertical pipe

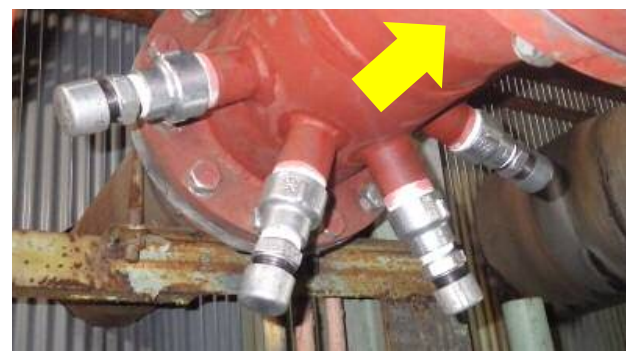

(b) Horizontal pipe

Fig. 5 Outline of sample point

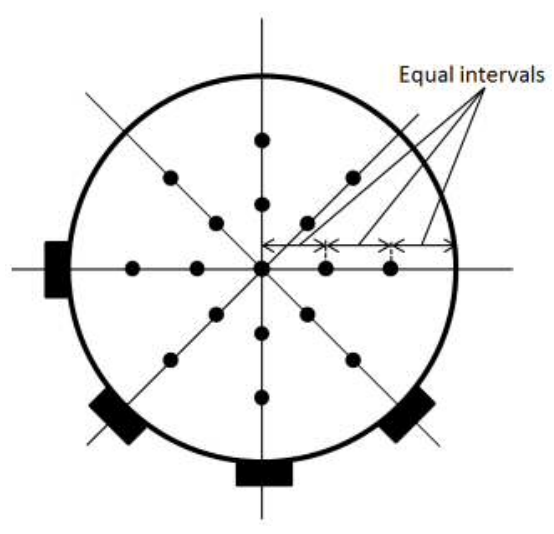

(a) Sampling station of equal areas
Table 1 Dimension of sampling tube tip

\begin{tabular}{|c|c|c|}
\hline $\begin{array}{c}\text { Sampling tube } \\
\text { No. }\end{array}$ & $\begin{array}{c}\text { Tip opening size } \\
(\mathrm{a} \times \mathrm{b})^{*}\end{array}$ & Remarks \\
\hline 1 & $20.5 \times 9.5$ & According to ASME PTC 4.2 1991. \\
\hline 2 & $20.5 \times 14.0$ & \\
\hline 3 & $20.5 \times 18.0$ & \\
\hline
\end{tabular}

*Dimension $a$ and b: Refer to Fig. 6

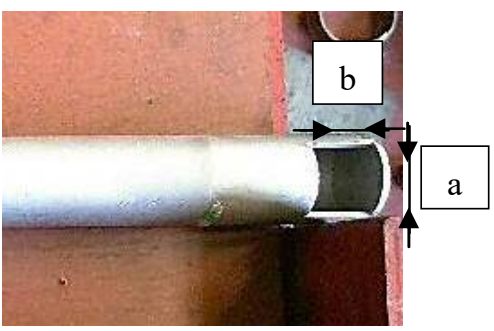

Fig. 6 Size of tip opening

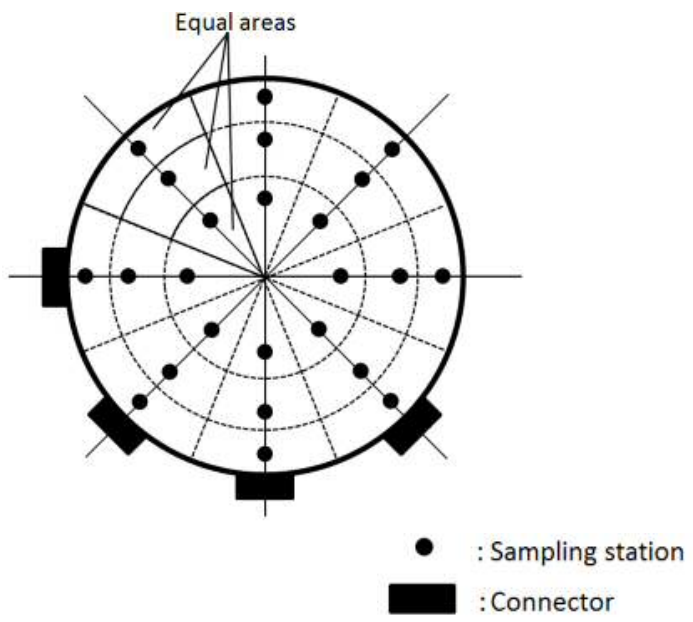

(b) Sampling station of equal intervals

Fig. 7 Sampling station on pipe cross-section

\section{Result}

\subsection{Distribution of woody biomass particles in the pipe cross-section}

The distribution of woody biomass particles in the vertical pipe is shown in Fig. 8 (a), and the distribution of woody biomass particles in the horizontal pipe at the sampling station is shown in Fig. 8 (b). The range inside each line (solid line, broken line, dashed-dotted line) and of light red in Fig. 8 (a), (b) is the place where the weight ratio is more than 1.5 times the average value (e.g. Fig. 8 (a): 100\% $\div 17$ (number of sampling stations) $=5.9 \%$ ), when woody 
biomass particles are distributed equally. The weight rate was calculated using the following procedure.

(1) Woody biomass particles are classified at each sampling station into 3 groups using 2 sieves (1 mm, $2 \mathrm{~mm})$ and the weight is measured for each.

(2) The same group weights were aggregated at all sampling stations.

(3) The weight of each group of each sampling station is divided by the value obtained in item (2) to obtain a weight ratio.

From the results in Fig. 8 (a) and (b), when the woody biomass particles size is different, the tendency of its presence range in the pipe also differs in the vertical and horizontal pipe.

Figure 9 shows the particle size distribution on a Rosin-Rammler diagram of woody biomass particles using two sample connections placed at a $90^{\circ}$ pitch or using four a sample connections placed at a $45^{\circ}$ pitch. Also, sampling tube No. 3 was used in the vertical pipe with a sampling time of $30 \mathrm{sec}$.. As a result, we confirmed a difference of around 5 to $6 \%$ for the rate when passing through a $1 \mathrm{~mm}$ sieve between using sample connections No. 1 and 3 , and sample connections No. 2 and 4.

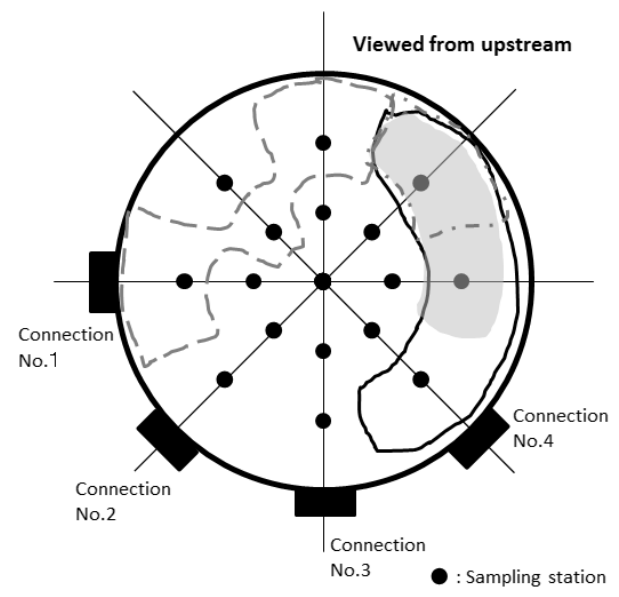

(a) Vertical pipe

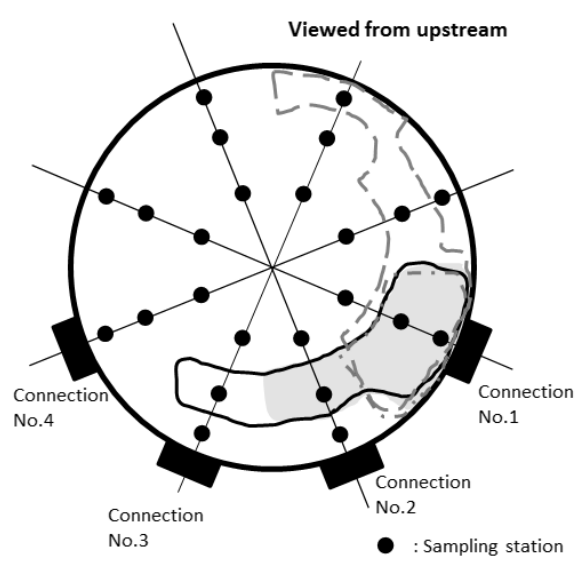

(b) Horizontal pipe

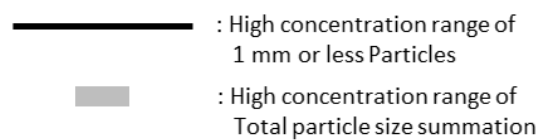

Fig. 8 Distribution of woody biomass particles in the pipe cross-section

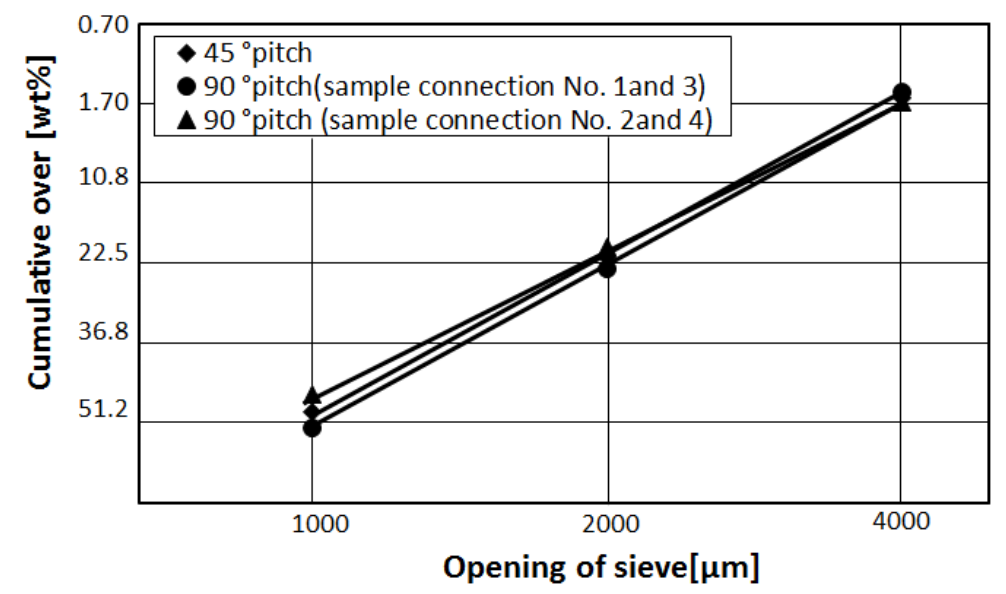

Fig. 9 Influence of sample connection pitch on particle size distribution on a Rosin-Rammler diagram 


\subsection{Sampling tube tip dimension}

Figure 10 shows the particle size distribution on a Rosin-Rammler diagram of woody biomass particles sampled with three types of sampling tube. These sampling tubes were used in the vertical pipe with a sampling time of 30 sec. As a result, it was confirmed that there is a difference of about 5 to $6 \%$ for the rate when passing through a $1 \mathrm{~mm}$ sieve.

\subsection{Sampling time per sampling station}

Figure 11 shows the particle size distribution on a Rosin-Rammler diagram of woody biomass particles sampled by the different sampling time per sampling station. Sampling tube No. 3 was used due to the possibility that large particles cannot be sampled if the opening of the Sampling tube tip is too small. That is why sampling tube No.3 was used in the vertical pipe, as it had the largest opening area of the tubes prepared this time. From Section 3.2, differences in sampling tubes (No.1 -3) does not significantly affect the particle size distribution.

As a result, it was confirmed that there is no difference in particle size other than $4 \mathrm{~mm}$. The variation at $4 \mathrm{~mm}$ is larger than other sample procedures.

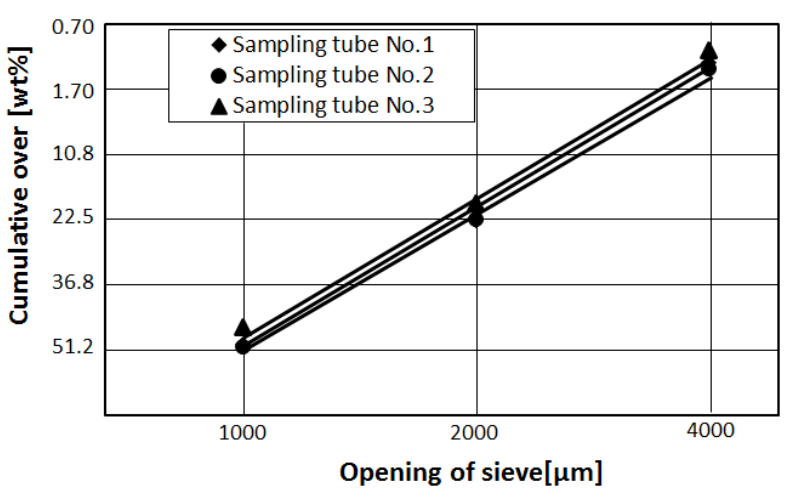

Fig. 10 Influence of sampling tube type on particle size distribution on a Rosin-Rammler diagram

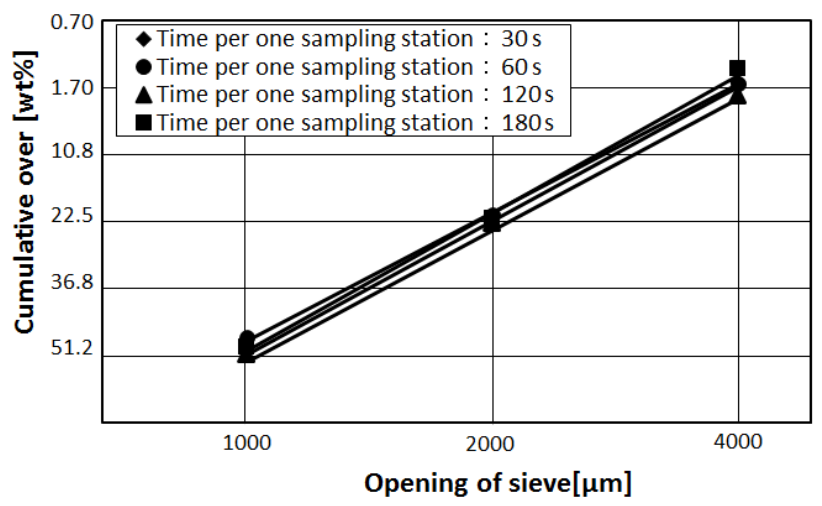

Fig. 11 Influence of sampling time per sampling station on particle size distribution on a Rosin-Rammler diagram

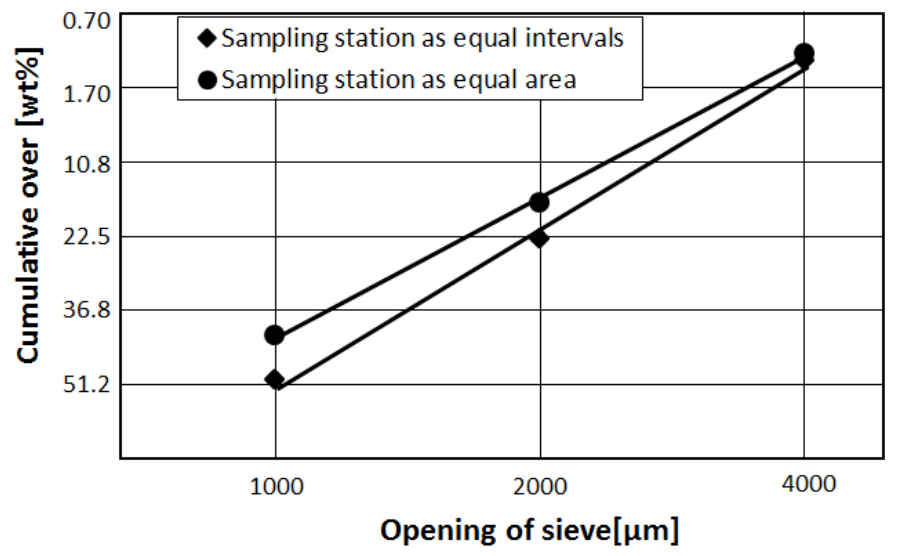

Fig. 12 Influence of location of sampling station on particle size distribution on a Rosin-Rammler diagram

\subsection{Sampling station}

The particle size distributions on a Rosin-Rammler diagram for the two sampling station arrangements are shown in Fig. 12. Sampling tube No. 3 was used at equal intervals and sampling tube No. 1 was used at equal areas. Sampling time was $30 \mathrm{sec}$. per sampling station. The sample point is vertical in each tube. Collection results are influenced by differences in sampling station. On the other hand differences in sampling tubes are considered to relatively little affect impact on result (Fig.12).

As a result, it was confirmed that there is a difference of about $8 \%$ for the rate when passing through a $1 \mathrm{~mm}$ sieve. 


\section{Discussion}

\subsection{Non-uniform flow of woody biomass particles in fuel pipe}

From Fig. 8 (a) and (b), it can be surmised that woody biomass particles of $1 \mathrm{~mm}$ or less flow in the pipe with the behavior shown in Fig. 13. Fig.13 is a diagram showing the fuel piping route between "Pulverizer "and "Exhaust fan" in Fig. 1 in detail. Woody biomass particles are expected to be passed outside of the elbow by the influence of centrifugal force. Furthermore, it is predicted that, once the flow becomes non-uniform, it will remain non-uniform downstream.

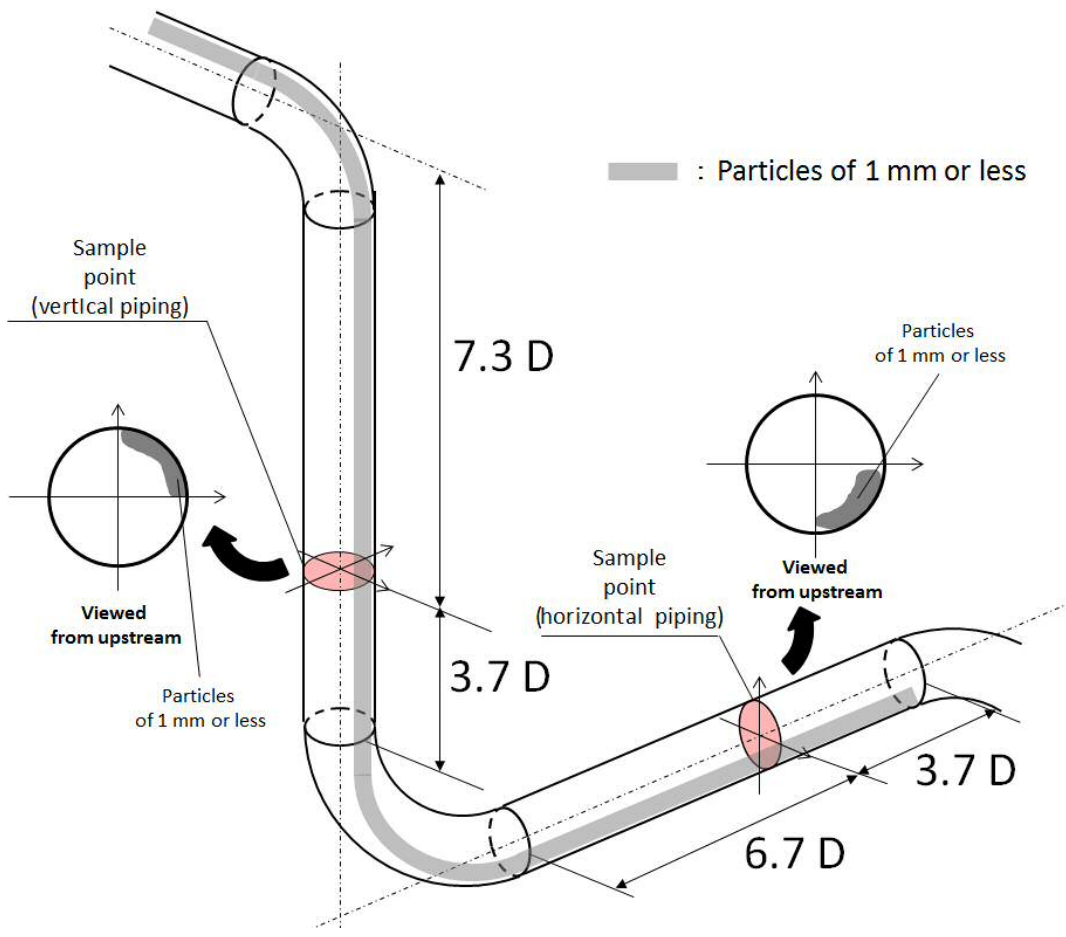

Fig. 13 Image of grinded woody pellet flow in fuel pipe

\subsection{Evaluation using coefficient of variation}

Large size woody biomass particles greatly influence combustibility in the boiler. Therefore, whether or not large size particles can be collected is an important point for sampling. In the steps described earlier, we used the rate of sieve passing. However, in this section, we use rate of sieve residue to focus on large size woody biomass particles.

The true value of the particle size distribution can be obtained when all the woody biomass particles have been collected and mixed evenly in the fuel pipe. In this test, it is difficult to obtain the true value of the particle size distribution, as not all woody biomass particles can be collected. Therefore, it is not known which sampling procedure is accurate, so we evaluate sampling procedure by the coefficient of the variation. The coefficient of variation is calculated by equation (1). The calculated value is evaluated on the basis of Table 2 . We calculate the coefficient of variation by average value and the standard deviation using the rate of sieve residue obtained by each sampling procedure.

When the coefficient of variation is larger, it means that a parameter having a larger difference from the true value is included. Thus, it can be determined that the collection result is influenced by the parameter.

When the coefficient of variation is smaller, it means that a parameter having a larger difference from the true value is not included. Thus, it can be judged that the parameter does not influence the collection result.

$$
V_{c}=\sigma \div \text { Ave }
$$

$V_{c}$ : Coefficient of variation

$\sigma$ : Standard deviation of sieve residue rate

Ave: Average of sieve residue rate 
Table 3 shows the combination of sampling procedure used to calculate the coefficient of variation. Six combinations of sampling procedure for calculation are shown as Case 1-6. In the Table 3, circles (O) indicate a sampling procedure used to calculate the coefficient of variation, and crosses $(\mathrm{x})$ indicates a sampling procedure not used. The explanation for each case is shown below. (An example of the method of calculating the coefficient of fluctuation relative to woody biomass of $4 \mathrm{~mm}$ or more in Case 2: After obtaining the standard deviation and average for the 9 woody biomass particles with a rate of $4 \mathrm{~mm}$ or more acquired in Procedure No. 3 through 11, apply to equation (1) and calculate.)

Case 1: The coefficient of variation is calculated using all sampling procedures.

Case 2: The coefficient of variation is calculated excluding the sampling procedure using sampling tubes No. 1 and No. 2 .

Case 3: The coefficient of variation is calculated excluding the sampling procedure with sampling times of 60,120 and $180 \mathrm{sec}$.

Case 4: The coefficient of variation is calculated excluding the sampling procedure at a $90^{\circ}$ pitch, at the horizontal sampling point.

Case 5: The coefficient of variation is calculated excluding the sampling procedure at a $90^{\circ}$ pitch.

Case 6: The coefficient of variation is calculated excluding the sampling procedure at a sampling station of equal area.

Table 2 Criteria of the variation

\begin{tabular}{|c|c|}
\hline Coefficient & Variation \\
\hline $\mathrm{x} \leq 0.2$ & Unacceptable \\
\hline $0.2<\mathrm{x} \leq 0.5$ & Miserable \\
\hline $0.5<\mathrm{x} \leq 1.0$ & Meritorious \\
\hline $1.0<\mathrm{x}$ & Marvelous \\
\hline
\end{tabular}

Table 3 Combination of sampling procedure used to calculate the coefficient of variation

\begin{tabular}{|c|c|c|c|c|c|c|c|c|c|c|c|}
\hline $\begin{array}{l}\text { Proced } \\
\text { ure No. }\end{array}$ & $\begin{array}{l}\text { Sampling } \\
\text { tube No. }\end{array}$ & $\begin{array}{l}\text { Sampling } \\
\text { Time[s] }\end{array}$ & $\begin{array}{c}\text { Sampling station } \\
\text { (A / I) } \\
\text { (equal "Area" or } \\
\text { "Interval") } \\
\end{array}$ & $\operatorname{Pitch}\left[{ }^{\circ}\right]$ & $\begin{array}{c}\text { Sample Point } \\
\text { (H / V) } \\
\text { ("Horizontial" or } \\
\text { "Vertical") } \\
\end{array}$ & Case 1 & Case 2 & Case 3 & Case 4 & Case 5 & Case 6 \\
\hline 1 & 1 & 30 & I & 45 & V & 0 & $\times$ & 0 & $\bigcirc$ & 0 & 0 \\
\hline 2 & 2 & 30 & I & 45 & V & $\bigcirc$ & $\times$ & $\bigcirc$ & $\bigcirc$ & $\bigcirc$ & $\bigcirc$ \\
\hline 3 & 3 & 30 & I & 45 & $\mathrm{~V}$ & 0 & 0 & 0 & 0 & 0 & 0 \\
\hline 4 & 3 & 60 & I & 45 & V & $\bigcirc$ & 0 & $\times$ & 0 & 0 & 0 \\
\hline 5 & 3 & 120 & I & 45 & V & $\bigcirc$ & $\bigcirc$ & $\times$ & $\bigcirc$ & $\bigcirc$ & 0 \\
\hline 6 & 3 & 180 & I & 45 & $\mathrm{~V}$ & 0 & 0 & $\times$ & 0 & 0 & 0 \\
\hline 7 & 3 & 30 & A & 45 & V & $\bigcirc$ & 0 & 0 & $\bigcirc$ & 0 & $\times$ \\
\hline $8 * 1$ & 3 & 30 & I & 90 & $\mathrm{~V}$ & 0 & 0 & 0 & 0 & $\times$ & 0 \\
\hline $9 *_{2}$ & 3 & 30 & I & 90 & $\mathrm{~V}$ & 0 & 0 & $\bigcirc$ & $\bigcirc$ & $x$ & 0 \\
\hline $10 *_{1}$ & 1 & 30 & A & 90 & $\mathrm{H}$ & 0 & 0 & 0 & $x$ & $x$ & $x$ \\
\hline $11 *_{2}$ & 1 & 30 & A & 90 & $\mathrm{H}$ & 0 & 0 & 0 & $\times$ & $\times$ & $\times$ \\
\hline
\end{tabular}

*1 Collected at sample Connection No. 1 and 3

*2 Collected at sample Connection No. 2 and 4

The coefficient of variation for each case is shown in Fig.14.

In Case 2, there is no significant difference in coefficient of variation with Case 1 at each sieve's residue size. Thus, the sampling tube tip may not influence the collected results.

In Cases 3 and 6, the coefficient of variation at a rate of $4 \mathrm{~mm}$ sieve residue differs to Case 1 . In Case 4 , the coefficient of variation at rate of $1 \mathrm{~mm} / 2 \mathrm{~mm}$ sieve residue differ to Case 1 .

The result obtained in Case 3 shows that the coefficient of variation at rate of $4 \mathrm{~mm}$ sieve residue differs to Case 1 . Thus, sampling time may influence the collected results. The detailed cause is unknown, but the sampling time may be too small. 
Moreover, Case 4, the coefficient of variation at a rate of $1 \mathrm{~mm} / 2 \mathrm{~mm}$ sieve residue, and Case 6 , the coefficient of variation at rate of $4 \mathrm{~mm}$ sieve residue, both differ to Case 1. Thus, sampling point and sampling station may also influence the collected results.

As mentioned above, the true result cannot be known unless all the woody biomass particles in the fuel pipe are collected. Although the correct collection method cannot be identified from this result, the three sampling parameters of sampling time, sample point and sampling station shall be the same, because the variation is large and a result collected using one method cannot be compared to a result collected using another method.

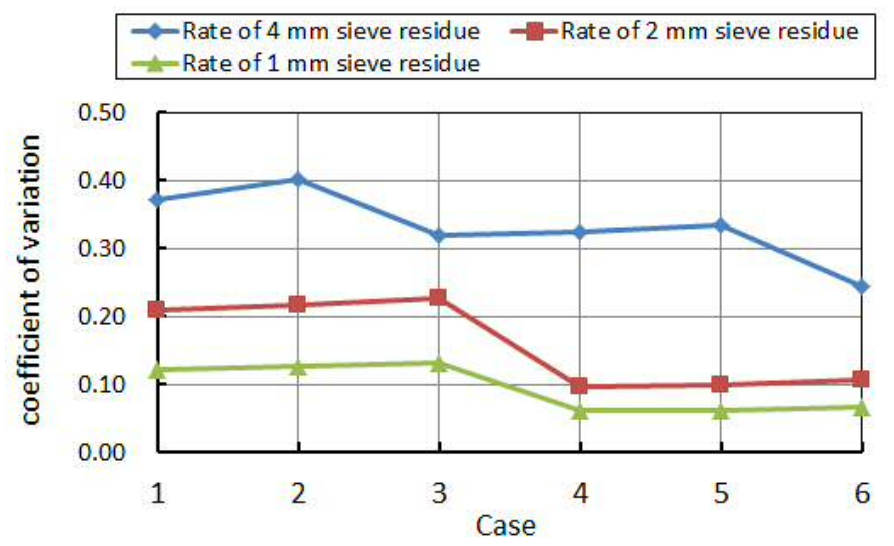

Fig. 14 Coefficient of variation for each case

\subsection{Sampling weight}

In ASME PTC 4.2 1991, it is a normal result if the value of equation (4) is between 0.9 and 1.1. Table 4 shows the results of comparing the theoretical collection amount and the actual collection amount of each sampling tube. From the results of Table 4, only sampling tube No. 1 has a rate within range.

Section 4.2 shows that the size of the sampling tip has minimal influence over the woody biomass particles size distribution collected. Thus, using sampling tubes No. 2 and 3 for the purpose of confirming the woody biomass particles size distribution, is considered non-problematic, as only the amount of collection increases. However, it is desirable to use sampling tube No. 1, because it is in the range of rate described in ASME.

The reason why the collection amount of sampling tubes No. 2 and 3 is larger than that of sampling tube No. 1 will be considered. In the range where woody biomass particles have a non-uniform flow, more woody biomass particles are collected when the tip opening area is large than when small. Therefore, it is considered that the collection amount will be large compared with the theoretical amount of woody biomass particles calculated, assuming that the woody particles flow uniformly.

Table 4 Comparison of sample weight

\begin{tabular}{|c|c|c|c|c|}
\hline $\begin{array}{c}\text { Sampling } \\
\text { tube No. }\end{array}$ & $\begin{array}{c}\text { Tip area } \\
{\left[\mathrm{mm}^{2}\right]}\end{array}$ & $\begin{array}{c}\text { Actual sample } \\
\text { weight }[\mathrm{g}]\end{array}$ & $\begin{array}{c}\text { Theoretical sample } \\
\text { weight }[\mathrm{g}]^{* 1}\end{array}$ & Rate*2 \\
\hline 1 & 195 & 1870 & 1926 & 1.03 \\
\hline 2 & 287 & 2479 & 2834 & 1.14 \\
\hline 3 & 369 & 3035 & 3644 & 1.20 \\
\hline
\end{tabular}

*1: Calculated by equation (3)

$* 2$ : Calculated by equation (4)

$$
W_{T}=\left(W_{F} \times S_{T}\right) \div\left(60 \times S_{F}\right)
$$

$W_{T}[\mathrm{~kg} / \mathrm{min}]$ : Theoretical value of amount of woody biomass particles collected

$W_{F}[\mathrm{~kg} / \mathrm{h}]: \quad$ Pellet feed rate to the mill

$S_{T}\left[\mathrm{~m}^{2}\right]: \quad$ Sampling tube tip

$S_{F}\left[\mathrm{~m}^{2}\right]: \quad$ Cross-section of fuel pipe 


$$
M_{T}=1000 \times W_{T} \times T_{S} \times n \div 60
$$

$M_{T}[\mathrm{~g}]$ : $\quad$ Theoretical amount of collected woody particles

$T_{S}$ [sec.] : $\quad$ Sampling time

$n$ [place] : $\quad$ Number of sampling stations

$$
\text { Rate }=M_{A} \div M_{T}
$$

$M_{A}[\mathrm{~g}]: \quad \quad$ Actual amount of collected woody biomass particles

\section{Conclusion}

(1) Confirmation of distribution of woody biomass particles in the fuel pipe cross-section Even when the $45^{\circ}$ pitch and the $90^{\circ}$ pitch were compared, the difference in the value of the coefficient of variation was small. However, because the flow in the piping is non-uniform, it may be better to use a $45^{\circ}$ pitch to obtain more accurate results.

(2) Effect of tip opening size of sampling tube Rate of particle size distribution remained the same even when a different sampling tube was used. However, it is desirable to use sampling tube No. 1 as the theoretical collection amount and actual collection amount value described in ASME are the closest.

(3) Investigation of sampling time

The variation in results increases if the measurement time differs. While the precise cause is unknown, it may be due to the sampling time being too small.

If the sampling time is the same, the variation in the collection result is small.

We consider that it is desirable to use 30 seconds, which is the shortest in this test, from the viewpoint of variability of results and workability.

When comparing other results, it is necessary to use the same arrangement of sampling time.

(4) Comparison of size distribution on arrangement of sampling station

In the pipe diameter in this test, the collection results have difference with the arrangement of two sampling stations. However, if the sampling station is the same, the variation in the collection result is small.

When comparing other results, it is necessary to use the same sampling station arrangement.

\section{References}

Agency for Natural Resources and Energy, Strategic Energy Plan (July, 2018)

America Society for Testing and Materials, "Standard Test Method for Sampling and Fineness Test of Pulverized Coal", ASTM D197 - 30, 1946

Feed-in tariff overview, Agency for Natural Resources and Energy, https://www.enecho.meti.go.jp/category/saving_and_new/saiene/kaitori/surcharge.html (accessed 2020-4-24)

IHI Corporation, Co-firing with a high-ratio of woody biomass to make the most of existing coal-fired thermal power generation systems, IHI Engineering Review Vol. 50 No.1 (2017)

The American Society of Mechanical Engineers United Engineering Center, "ASME PERFORMANCE TEST CODES Test Code for COAL PULVERIZERS.” , ASME PTC 4.2, pp.18-25, 1991 